\title{
Adomian Decomposition Method for Approximating the Solution of the High-Order Dispersive Cubic-Quintic Nonlinear Schrödinger Equation
}

\author{
Xian-Jing Lai ${ }^{\mathrm{a}}$, Jie-Fang Zhang ${ }^{\mathrm{b}}$, and Jian-Fei Luo ${ }^{\mathrm{c}}$ \\ ${ }^{a}$ Department of Basic Science, Zhejiang Shuren University, Hangzhou, 310015, Zhejiang, China \\ ${ }^{\mathrm{b}}$ Institute of Theoretical Physics, Zhejiang Normal University, Jinhua, 321004, Zhejiang, China \\ ${ }^{c}$ President's Office, Zhejiang Normal University, Jinhua, 321004, Zhejiang, China \\ Reprint requests to X.-J.L.; E-mail: laixianjing@163.com
}

Z. Naturforsch. 61a, 205 - 215 (2006); received December 20, 2005

In this paper, the decomposition method is implemented for solving the high-order dispersive cubic-quintic nonlinear Schrödinger equation. By means of Maple the Adomian polynomials of obtained series solution have been calculated. The results reported in this article provide further evidence of the usefulness of Adomain decomposition for obtaining solutions of nonlinear problems. PACS numbers: 02.30.Jr; 02.60.Cb; 42.65.Tg

Key words: Adomian Decomposition Method; High-Order Dispersive Cubic-Quintic Nonlinear Schrödinger Equation; Adomian Polynomials.

\section{Introduction}

The dynamics of solitons in Kerr media are in general described by the nonlinear Schrödinger (NLS) family of equations with cubic nonlinear terms [1-5]. However, when the intensity of the incident light field gets stranger and stranger, one can not neglect the non-Kerr nonlinearity effects and NLS equations with higher-order dispersion terms are needed to describe the propagation of optical pulses in fibers $[6,7]$. The general higher-order nonlinear Schrödinger (HONLS) equation models proposed in the literature are not completely integrable and cannot be exactly solved by the inverse scattering transform method [8]. The nonintegrability usually originates not only from the higherorder nonlinear terms but also the higher dispersion terms. The analytical and numerical solutions for the NLS equations with higher nonlinearity and dispersion have been actively investigated by many authors in several different models [6-11].

In this paper we consider a higher-order NLS equation including fourth-order dispersion with a parabolic nonlinearity law. This high dispersive cubic-quintic nonlinear Schrödinger (HDCNS) equation can be written in the form

$\mathrm{i} \Psi_{z}=\frac{\beta_{2}}{2} \Psi_{t t}+\mathrm{i} \frac{\beta_{3}}{6} \Psi_{t t t}+\frac{\beta_{4}}{24} \Psi_{t t t t}-\gamma_{1}|\Psi|^{2} \Psi-\gamma_{2}|\Psi|^{4} \Psi$, with the initial condition

$$
\Psi(t, 0)=f(t)
$$

where $\mathrm{i}=\sqrt{-1}, \Psi(t, z)$ is the slowly varying envelope of the electromagnetic field, $t$ represents the time (in the group-velocity frame), $z$ represents the distance along the direction of propagation (the longitudinal coordinate), $\beta_{2}, \beta_{3}$ and $\beta_{4}$ represent coefficients of second-order dispersive (GVD), third-order dispersive (TOD), fourth-order dispersive (FOD), respectively, and $\gamma_{1}$ and $\gamma_{2}$ are coefficients of the cubic and quintic nonlinearities, respectively. In [12] the modulational instability of optical waves to (1) is investigated. For picosecond light pulses, the higher-order terms of (1) can be omitted, i.e., $\beta_{3}=\beta_{4}=\gamma_{2}=0$, and (1) can reduce to the NLS equation. The NLS equation including only the GVD and the self-phase modulation (SPM) is well known in the fiber, and it admits bright and dark soliton-type pulse propagation in anomalous and normal dispersion regimes, respectively. However, for femtosecond light pulses, whose duration is shorter than $100 \mathrm{fs}$, the higher-order terms are nonnegligible and should be retained. When $\beta_{3}=\beta_{4}=0$ in (1), it was shown that the dark and bright solitary wave solutions exist even in the normal dispersion regime. Recently, dark and bright soliton solutions have been proposed for (1) with third-order dispersion being nil $\left(\beta_{3}=0\right)$ [13]. Besides, in the absence of the quintic 
nonlinear term $\left(\gamma_{2}=0\right)$ of $(1)$, Shagalov [14] has investigated the effect of the third- and fourth-order dispersion terms on the modulational instability, and Karpman and Shagalov [15-16] have studied the time behavior of the amplitudes, velocities and other parameters of radiating solitons.

For (1) with both the TOD and FOD and the cubicquintic nonlinear terms, an ordinary soliton moving with constant velocity $V_{0}$ (in application to the nonlinear optical pulses having constant self-frequency shift) has the form

$$
\Psi=\phi\left(z-z_{0}-V_{0} t\right) \exp \left(\mathrm{i}\left[\eta\left(z-V_{0} t\right)+\sigma t+\varphi_{0}\right]\right),
$$

where $V_{0}$ is a soliton velocity, $\eta$ a soliton wave-number and $\sigma$ a nonlinear frequency shift. Arbitrary constants $z_{0}$ and $\varphi_{0}$ will be omitted below for brevity. In particular, a solution with an amplitude that only depends on the time and a phase only depending on the coordinate in the direction of propagation such as [11]

$$
\Psi=\lambda_{0} \tanh \left(-V_{0} t\right) \exp (\mathrm{i} \eta z),
$$

where $\lambda_{0}, v=-1 / V_{0}$ and $\eta$ are the parameters representing the amplitude, pulsewidth and wavevector per unit length, respectively, is to be determined as

$$
\begin{aligned}
& \lambda_{0}=\frac{1}{5} \sqrt{-\frac{15}{\gamma_{2}}\left(\gamma_{1}-\frac{\beta_{2}}{\sqrt{-\beta_{4} / \gamma_{2}}}\right)}, \\
& V_{0}=-3 \sqrt{\frac{-\beta_{2}+\gamma_{1} \sqrt{-\beta_{4} / \gamma_{2}}}{15 \beta_{4}}}, \\
& \eta=\frac{3\left(-\beta_{2}+\gamma_{1} \sqrt{-\beta_{4} / \gamma_{2}}\right)\left(3 \beta_{2}+2 \gamma_{1} \sqrt{-\beta_{4} / \gamma_{2}}\right)}{25 \beta_{4}} .
\end{aligned}
$$

It is worthwhile to investigate the numerical solutions (in particular soliton solutions) for the HDCNS equation. In this paper, we aim to introduce a reliable algorithm, the Adomian decomposition method (ADM), to approach (1) with initial profile. Until now, several studies in the literature have been conducted to implement ADM to the NLS equation [17-23]. ADM is a numerical technique for solving a wide class of linear or nonlinear, algebraic or ordinary/partial differential equations. The method, which is well addressed in [24-26], has a useful attraction in that it provides the solution as an infinite series in which each term can be easily determined. The series is quickly convergent towards an accurate solution. It has been proved to be a competitive alternative to the Taylor series method and other series techniques. Several papers deal with the comparison of the ADM with some existing techniques in solving different types of problems. In [27], it was found that, unlike other series solution methods, the decomposition method is easy to program in engineering problems, and provides immediate and visible solution terms without linearization and discretization. Advantages of the ADM over the Picard's method have been proved by Rach [28]. He showed that the two methods are not the same and the Picard's method works only if the equation satisfies the Lipschitz condition. Edwards et al. [29] have introduced their comparison of the ADM and Runge-Kutta methods for approximate solutions of some predator prey model equations. Wazwaz introduced a comparison between the ADM and Taylor series method [30]; he showed that the ADM minimizes the computational difficulties of the Taylor series in that the components of the solution are determined elegantly by using simple integrals, although the Taylor series method provides the same answer obtained by ADM. In [31] the ADM and wavelet-Galerkin method is compared. From the computational viewpoint, the comparison shows that the ADM is efficient and easy to use. In [32], a comparison of the numerical results is obtained by using the B-spline finite element method and ADM. From the results, the ADM algorithm provides highly accurate numerical solutions without spatial discretizations for the nonlinear partial differential equation. The illustrations show that the ADM is numerically more accurate than the conventional numerical method of the finite element. Subsequent works in this direction have demonstrated the power of the method for numerical evaluations.

\section{The Method of Solution}

This section is devoted to review the ADM for solving the HDCNS equation with the initial condition $\Psi(t, 0)=f(t)$. Following the Adomian decomposition analysis, we rewrite (1) in the following operator form:

$$
\begin{aligned}
L_{z} \Psi= & -\mathrm{i} \frac{\beta_{2}}{2} L_{2, t} \Psi+\frac{\beta_{3}}{6} L_{3, t} \Psi-\mathrm{i} \frac{\beta_{4}}{24} L_{4, t} \Psi \\
& +\gamma_{1}|\Psi|^{2} \Psi+\gamma_{2}|\Psi|^{4} \Psi .
\end{aligned}
$$

Similar to [17-19], we define for (1) the linear operators $L_{z} \equiv \frac{\partial}{\partial z}, L_{2, t} \equiv \frac{\partial^{2}}{\partial t^{2}}, L_{3, t} \equiv \frac{\partial^{3}}{\partial t^{3}}$ and $L_{4, t} \equiv \frac{\partial^{4}}{\partial t^{4}}$. By defining the onefold right-inverse operator $L_{z}^{-1} \equiv$ 
$\int_{0}^{z}(\cdot) \mathrm{d} z$, we find that

$$
\begin{aligned}
\Psi(t, z)= & \Psi(t, 0)+L_{z}^{-1}\left[-\mathrm{i} \frac{\beta_{2}}{2} L_{2, t} \Psi+\frac{\beta_{3}}{6} L_{3, t} \Psi\right. \\
& \left.-\mathrm{i} \frac{\beta_{4}}{24} L_{4, t} \Psi+\gamma_{1}|\Psi|^{2} \Psi+\gamma_{2}|\Psi|^{4} \Psi\right]
\end{aligned}
$$

Therefore

$$
\begin{aligned}
\Psi(t, z)= & f(t)+L_{z}^{-1}\left[-\mathrm{i} \frac{\beta_{2}}{2} L_{2, t} \Psi+\frac{\beta_{3}}{6} L_{3, t} \Psi\right. \\
& \left.-\mathrm{i} \frac{\beta_{4}}{24} L_{4, t} \Psi+\gamma_{1} G_{1}(\Psi)+\gamma_{2} G_{2}(\Psi)\right] .
\end{aligned}
$$

The decomposition method suggests that the linear terms $\Psi(t, z)$ be decomposed by an infinite series of components

$$
\Psi(t, z)=\sum_{n=0}^{\infty} \Psi_{n}(t, z)
$$

where the components $\Psi_{0}, \Psi_{1}, \Psi_{2}, \ldots$, as will be seen later, are to be determined individually in an easy way through a recursive relation that involves simple integrals. The nonlinear operators $G_{1}(t, z)$ and $G_{2}(t, z)$ are defined by the infinite series

$$
G_{i}(\Psi)=\sum_{n=0}^{\infty} A_{i, n}, \quad i=1,2 .
$$

That means that the nonlinear terms $|\Psi|^{2} \Psi$ and $|\Psi|^{4} \Psi$ are represented series of $A_{i, n},(i=1,2)$ which are called Adomian polynomials. In next $\Psi_{n}(t, z),(n \geq 0)$ is the component of $\Psi(t, z)$ that will elegantly be determined. Hence, upon substituting these decomposition series into (5) yields

$$
\begin{aligned}
& \sum_{n=0}^{\infty} \Psi_{n}(t, z)=f(t) \\
& -\left[\mathrm{i} \frac{\beta_{2}}{2} \sum_{n=0}^{\infty} L_{z}^{-1} L_{2, t} \Psi_{n}(t, z)+\frac{\beta_{3}}{6} \sum_{n=0}^{\infty} L_{z}^{-1} L_{3, t} \Psi_{n}(t, z)\right. \\
& \left.\quad-\mathrm{i} \frac{\beta_{4}}{24} \sum_{n=0}^{\infty} L_{z}^{-1} L_{4, t} \Psi_{n}(t, z)+\gamma_{1} \sum_{n=0}^{\infty} A_{1, n}+\gamma_{2} \sum_{n=0}^{\infty} A_{2, n}\right] .
\end{aligned}
$$

The method suggests that the zeroth component $\Psi_{0}$ is usually defined as the terms arising from initial conditions. Then we obtain the components series solution by the following recursive relationship:

$$
\Psi_{0}(t, 0)=f(t)
$$

$$
\begin{aligned}
\Psi_{n+1}=L_{z}^{-1}[ & -i \frac{\beta_{2}}{2} L_{2, t} \Psi_{n}+\frac{\beta_{3}}{6} L_{3, t} \Psi_{n} \\
& \left.-\mathrm{i} \frac{\beta_{4}}{24} L_{4, t} \Psi_{n}+\gamma_{1} A_{1, n}+\gamma_{2} A_{2, n}\right],
\end{aligned}
$$

where $n \geq 0$.

The Adomian polynomials $A_{i, n}$ can be generated for all forms of nonlinearity which are generated according to the following algorithm:

$$
A_{i, n}=\frac{1}{n !}\left[\frac{\mathrm{d}^{n}}{\mathrm{~d} \alpha^{n}} G_{i}\left(\sum_{k=0}^{n} \alpha^{k} \Psi_{k}\right)\right]_{\alpha=0}, n \geq 0 .
$$

This formula is easy to be set in a computer code to get as many polynomials as we need in the calculation. We can give the first few Adomian polynomials of the $A_{i, n}$ as

$$
\begin{aligned}
A_{1,0}= & \left|\Psi_{0}\right|^{2} \Psi_{0} \\
A_{1,1}= & 2\left|\Psi_{0}\right|^{2} \Psi_{1}+\Psi_{0}^{2} \bar{\Psi}_{1} \\
A_{1,2}= & 2\left|\Psi_{0}\right|^{2} \Psi_{2}+\bar{\Psi}_{0} \Psi_{1}^{2}+2\left|\Psi_{1}\right|^{2} \Psi_{0}+\Psi_{0}^{2} \bar{\Psi}_{2} \\
A_{1,3}= & 2\left|\Psi_{0}\right|^{2} \Psi_{3}+2 \bar{\Psi}_{0} \Psi_{1} \Psi_{2}+2 \Psi_{0} \bar{\Psi}_{1} \Psi_{2} \\
& +\left|\Psi_{1}\right|^{2} \Psi_{1}+2 \Psi_{0} \Psi_{1} \bar{\Psi}_{2}+\Psi_{0}^{2} \bar{\Psi}_{3} \\
A_{1,4}= & 2 \Psi_{0}^{2} \bar{\Psi}_{4}+2 \bar{\Psi}_{0} \Psi_{1} \Psi_{3}+\left|\Psi_{2}\right|^{2} \bar{\Psi}_{0} \\
& +2 \Psi_{0} \bar{\Psi}_{1} \Psi_{3}+2\left|\Psi_{1}\right|^{2} \Psi_{2}+2\left|\Psi_{2}\right|^{2} \Psi_{0} \\
& +\Psi_{1}^{2} \bar{\Psi}_{2}+2 \Psi_{0} \Psi_{1} \bar{\Psi}_{3}+\Psi_{0}^{2} \bar{\Psi}_{4} \\
A_{2,0}= & \left|\Psi_{0}\right|^{4} \Psi_{0}, \\
A_{2,1}= & 3\left|\Psi_{0}\right|^{4} \Psi_{1}+2\left|\Psi_{0}\right|^{2} \Psi_{0}^{2} \bar{\Psi}_{1} \\
A_{2,2}= & 3\left|\Psi_{0}\right|^{2} \bar{\Psi}_{0} \Psi_{1}^{2}+6\left|\Psi_{0}\right|^{2} \Psi_{0}\left|\Psi_{1}\right|^{2} \\
& +3\left|\Psi_{0}\right|^{4} \Psi_{2}+\Psi_{0}^{3} \bar{\Psi}_{1}^{2}+2\left|\Psi_{0}\right|^{2} \Psi_{0}^{2} \bar{\Psi}_{2} \\
A_{2,3}= & \Psi_{1}^{3} \bar{\Psi}_{0}^{2}+6\left|\Psi_{0}\right|^{2}\left|\Psi_{1}\right|^{2} \Psi_{1}+6\left|\Psi_{0}\right|^{2} \bar{\Psi}_{0} \Psi_{1} \Psi_{2} \\
& +3 \Psi_{0}^{2}\left|\Psi_{1}\right|^{2} \bar{\Psi}_{1}+6\left|\Psi_{0}\right|^{2} \Psi_{0} \Psi_{2} \bar{\Psi}_{1} \\
& +6\left|\Psi_{0}\right|^{2} \Psi_{0} \Psi_{1} \bar{\Psi}_{2}+3\left|\Psi_{0}\right|^{4} \Psi_{3} \\
& +2 \Psi_{0}^{3} \bar{\Psi}_{1} \bar{\Psi}_{2}+2\left|\Psi_{0}\right|^{2} \Psi_{0}^{2} \bar{\Psi}_{3} \\
A_{2,4}= & 3 \Psi_{0}\left|\Psi_{1}\right|^{4}+6\left|\Psi_{0}\right|^{2} \Psi_{1}^{2} \bar{\Psi}_{2}+3\left|\Psi_{0}\right|^{2} \bar{\Psi}_{0} \Psi_{2}^{2} \\
+ & 6\left|\Psi_{0}\right|^{2} \bar{\Psi}_{0} \Psi_{1} \Psi_{3}+6 \Psi_{0}^{2}\left|\Psi_{1}\right|^{2} \bar{\Psi}_{2} \\
+ & 6\left|\Psi_{0}\right|^{2} \Psi_{0} \Psi_{3} \bar{\Psi}_{1}+6\left|\Psi_{0}\right|^{2} \Psi_{0}\left|\Psi_{2}\right|^{2} \\
+ & 6\left|\Psi_{0}\right|^{2} \Psi_{0} \Psi_{1} \bar{\Psi}_{3}+3 \Psi_{1}^{2} \bar{\Psi}_{0}^{2} \Psi_{2} \\
+ & 2\left|\Psi_{1}\right|^{2} \Psi_{1}^{2} \bar{\Psi}_{0}+3 \Psi_{0}^{2} \bar{\Psi}_{1}^{2} \Psi_{2} \\
+ & 3\left|\Psi_{0}\right|^{4} \Psi_{4}+\Psi_{0}^{3} \bar{\Psi}_{2}^{2} \\
+ & 2 \Psi_{0}^{3} \bar{\Psi}_{1} \bar{\Psi}_{3}+2\left|\Psi_{0}\right|^{2} \Psi_{0}^{2} \bar{\Psi}_{4} \\
+ & 12\left|\Psi_{0}\right|^{2}\left|\Psi_{1}\right|^{2} \Psi_{2} \\
A_{1} & \\
&
\end{aligned}
$$


The rest of the polynomials can be constructed in a similar manner. In case the nonlinear terms $G_{1}(\Psi)=$ $|\Psi|^{2} \Psi$ and $G_{2}(\Psi)=|\Psi|^{4} \Psi$ are real functions then the Adomian polynomials are evaluated by first writing

$$
|\Psi|=\Psi|H(\Psi)-H(-\Psi)|,
$$

where $H(u)$ is the Heaviside (step) function. Hence, (13) yields

$$
\begin{aligned}
& G_{1}(\Psi)=|\Psi|^{2} \Psi=\Psi^{3}[H(\Psi)-H(-\Psi)]^{2} \\
& G_{2}(\Psi)=|\Psi|^{4} \Psi=\Psi^{5}[H(\Psi)-H(-\Psi)]^{2}
\end{aligned}
$$

Therefore,

$$
\begin{aligned}
& G_{1}(\Psi)=\sum_{n=0}^{\infty}[H(\Psi)-H(-\Psi)]^{2} A_{1, n}^{\prime}\left\{\Psi^{3}\right\} \\
& G_{2}(\Psi)=\sum_{n=0}^{\infty}[H(\Psi)-H(-\Psi)]^{2} A_{2, n}^{\prime}\left\{\Psi^{5}\right\}
\end{aligned}
$$

where $A_{1, n}^{\prime}\left\{\Psi^{3}\right\}$ and $A_{2, n}^{\prime}\left\{\Psi^{5}\right\}$ are the Adomian polynomials given by

$$
\begin{aligned}
A_{1,0}^{\prime}= & \Psi_{0}^{3}, \quad A_{1,1}=3 \Psi_{0}^{2} \Psi_{1}, \\
A_{1,2}^{\prime}= & 3 \Psi_{0}^{2} \Psi_{2}+3 \Psi_{0} \Psi_{1}^{2} \\
A_{1,3}^{\prime}= & 3 \Psi_{0}^{2} \Psi_{3}+6 \Psi_{0} \Psi_{1} \Psi_{2}+\Psi_{1}^{3} \\
A_{1,4}^{\prime}= & 3 \Psi_{0}^{2} \Psi_{4}+6 \Psi_{0} \Psi_{1} \Psi_{3}+3 \Psi_{1}^{2} \Psi_{2}+3 \Psi_{2}^{2} \Psi_{0}, \\
A_{2,0}^{\prime}= & \Psi_{0}^{5}, \quad A_{2,1}=5 \Psi_{0}^{4} \Psi_{1}, \\
A_{2,2}^{\prime}= & 10 \Psi_{0}^{3} \Psi_{1}^{2}+5 \Psi_{0}^{4} \Psi_{2}, \\
A_{2,3}^{\prime}= & 10 \Psi_{1}^{3} \Psi_{0}^{2}+20 \Psi_{0}^{3} \Psi_{2} \Psi_{1}+5 \Psi_{0}^{4} \Psi_{3}, \\
A_{2,4}^{\prime}= & 5 \Psi_{0} \Psi_{1}^{4}+30 \Psi_{0}^{2} \Psi_{1}^{2} \Psi_{2}+10 \Psi_{0}^{3} \Psi_{2}^{2} \\
& +20 \Psi_{0}^{3} \Psi_{1} \Psi_{3}+5 \Psi_{0}^{4} \Psi_{4} .
\end{aligned}
$$

iablel with a singularity at the origin. Since $[H(\Psi)-$ $H(-\Psi)]^{2}=1$ for $\Psi \neq 0$, it follows from (14) that, if we avoid the origin, then $G_{1}(\Psi)=\Psi^{3}=\sum_{n=0}^{\infty} A_{1, n}^{\prime}$ and $G_{2}(\Psi)=\Psi^{5}=\sum_{n=0}^{\infty} A_{2, n}^{\prime}$

\section{Exemplification of the ADM}

We first consider the application of the decomposition method to the HDCNS equation with the initial condition

$$
\begin{aligned}
& \Psi(t, 0)= \\
& \lambda \operatorname{sech}\left(-\sqrt{\frac{\beta_{4} \lambda^{2}\left(5 \gamma_{2} \lambda^{2}-6 \gamma_{1}\right)}{3 \beta_{3}^{2}+6 \beta_{2} \beta_{4}}} t\right) \exp \left(-\mathrm{i} \frac{\beta_{3}}{\beta_{4}} t\right),
\end{aligned}
$$

where $\beta_{2}, \beta_{3}, \beta_{4}, \gamma_{1}, \gamma_{2}$ and $\lambda$ are arbitrary constants.

Applying the inverse operator $L_{z}^{-1}$ on both sides of (3) and using the initial condition (17) and the decomposition series (6) and (7) yields

$$
\begin{aligned}
& \sum_{n=0}^{\infty} \Psi_{n}(t, z)= \\
& \lambda \operatorname{sech}\left(-\sqrt{\frac{\beta_{4} \lambda^{2}\left(5 \gamma_{2} \lambda^{2}-6 \gamma_{1}\right)}{3 \beta_{3}^{2}+6 \beta_{2} \beta_{4}}} t\right) \exp \left(-\mathrm{i} \frac{\beta_{3}}{\beta_{4}} t\right) \\
& -\left[\mathrm{i} \frac{\beta_{2}}{2} \sum_{n=0}^{\infty} L_{z}^{-1} L_{2, t} \Psi_{n}(t, z)+\frac{\beta_{3}}{6} \sum_{n=0}^{\infty} L_{z}^{-1} L_{3, t} \Psi_{n}(t, z)\right. \\
& \left.-\mathrm{i} \frac{\beta_{4}}{24} \sum_{n=0}^{\infty} L_{z}^{-1} L_{4, t} \Psi_{n}(t, z)+\gamma_{1} \sum_{n=0}^{\infty} A_{1, n}+\gamma_{2} \sum_{n=0}^{\infty} A_{2, n}\right] .
\end{aligned}
$$

For simpleness, we take $\omega=\left[\beta_{4} \lambda^{2}\left(5 \gamma_{2} \lambda^{2}-6 \gamma_{1}\right) /\right.$ $\left.\left(3 \beta_{3}^{2}+6 \beta_{2} \beta_{4}\right)\right]^{1 / 2}$ in the following. Proceeding as before, the Adomian decomposition method gives the recurrence relation

$$
\Psi_{0}=\Psi(t, 0)=\lambda \operatorname{sech}(-\omega t) \exp \left(-\mathrm{i} \frac{\beta_{3}}{\beta_{4}} t\right),
$$$$
\Psi_{n+1}(t, z)=L_{z}^{-1}\left[-\mathrm{i} \frac{\beta_{2}}{2} L_{2, t} \Psi_{n}+\frac{\beta_{3}}{6} L_{3, t} \Psi_{n}-\mathrm{i} \frac{\beta_{4}}{24} L_{4, t} \Psi_{n}+\gamma_{1} A_{1, n}+\gamma_{2} A_{2, n}\right],
$$

where $n \geq 0$. The resulting components are

$$
\Psi_{0}=\lambda \operatorname{sech}(\omega t) \exp \left(-\mathrm{i} \frac{\beta_{3}}{\beta_{4}} t\right)
$$

$$
\Psi_{1}=L_{z}^{-1}\left[-\mathrm{i} \frac{\beta_{2}}{2} L_{2, t} \Psi_{0}+\frac{\beta_{3}}{6} L_{3, t} \Psi_{0}-\mathrm{i} \frac{\beta_{4}}{24} L_{4, t} \Psi_{0}+\gamma_{1} A_{1,0}+\gamma_{2} A_{2,0}\right]=\frac{z \lambda}{24 \cosh (\omega t)^{2} \beta_{4}^{3}}
$$$$
\text { . }\left[\mathrm{i}\left(3 \beta_{3}^{4}+12 \beta_{2} \beta_{3}^{2} \beta_{4}-\beta_{4}^{4} \omega^{4}-12 \beta_{2} \omega^{2} \beta_{4}^{3}-6 \beta_{3}^{2} \omega^{2} \beta_{4}^{2}\right) \cosh (\omega t)+\left(24 \beta_{3} \omega \beta_{4}^{2} \beta_{2}+8 \beta_{3}^{3} \omega \beta_{4}\right) \sinh (\omega t)\right] \exp \left(-\mathrm{i} \frac{\beta_{3}}{\beta_{4}} t\right),
$$ 


$$
\begin{aligned}
& \Psi_{2}=L_{z}^{-1}\left[-\mathrm{i} \frac{\beta_{2}}{2} L_{2, t} \Psi_{1}+\frac{\beta_{3}}{6} L_{3, t} \Psi_{1}-\mathrm{i} \frac{\beta_{4}}{24} L_{4, t} \Psi_{1}+\gamma_{1} A_{1,1}+\gamma_{2} A_{2,1}\right]=-\frac{z^{2} \lambda}{1152 \cosh (\omega t)^{3} \beta_{4}^{6}} \\
& \cdot\left[\left(\beta_{4}^{8} \omega^{8}+120 \beta_{2} \omega^{4} \beta_{3}^{2} \beta_{4}^{5}+144 \beta_{2}^{2} \beta_{3}^{4} \beta_{4}^{2}-100 \beta_{3}^{6} \omega^{2} \beta_{4}^{2}-864 \beta_{2}^{2} \beta_{3}^{2} \beta_{4}^{4} \omega^{2}+144 \beta_{2}^{2} \omega^{4} \beta_{4}^{6}+72 \beta_{2} \beta_{3}^{6} \beta_{4}\right.\right. \\
& \left.-600 \beta_{3}^{4} \beta_{2} \omega^{2} \beta_{4}^{3}+24 \beta_{4}^{7} \beta_{2} \omega^{6}+12 \omega^{6} \beta_{3}^{2} \beta_{4}^{6}+30 \beta_{3}^{4} \omega^{4} \beta_{4}^{4}+9 \beta_{3}^{8}\right) \cosh (\omega t)^{2} \\
& +\mathrm{i}\left(576 \beta_{2}^{2} \omega^{3} \beta_{3} \beta_{4}^{5}+16 \beta_{3}^{3} \omega^{5} \beta_{4}^{5}+48 \beta_{2} \omega^{5} \beta_{3} \beta_{4}^{6}+480 \beta_{2} \beta_{3}^{3} \omega^{3} \beta_{4}^{4}+96 \beta_{3}^{5} \omega^{3} \beta_{4}^{3}-48 \beta_{3}^{7} \omega \beta_{4}-336 \beta_{2} \beta_{3}^{5} \omega \beta_{4}^{2}\right. \\
& \left.\left.-576 \beta_{2}^{2} \omega \beta_{3}^{3} \beta_{4}^{3}\right) \sinh (\omega t) \cosh (\omega t)+1152 \beta_{2}^{2} \beta_{3}^{2} \beta_{4}^{4} \omega^{2}+128 \beta_{3}^{6} \omega^{2} \beta_{4}^{2}+768 \beta_{3}^{4} \beta_{2} \omega^{2} \beta_{4}^{3}\right] \exp \left(-\mathrm{i} \frac{\beta_{3}}{\beta_{4}} t\right), \\
& \Psi_{3}=L_{z}^{-1}\left[-\mathrm{i} \frac{\beta_{2}}{2} L_{2, t} \Psi_{1}+\frac{\beta_{3}}{6} L_{3, t} \Psi_{2}-\mathrm{i} \frac{\beta_{4}}{24} L_{4, t} \Psi_{2}+\gamma_{1} A_{1,2}+\gamma_{2} A_{2,2}\right]=-\frac{z^{3} \lambda}{82944 \cosh (\omega t)^{4} \beta_{4}^{9}} \\
& \text {. }\left[\mathrm { i } \left(-1728 \beta_{2}^{3} \omega^{6} \beta_{4}^{9}+84 \beta_{3}^{6} \omega^{6} \beta_{4}^{6}+27 \beta_{3}^{12}-99 \beta_{3}^{4} \beta_{4}^{8} \omega^{8}+1296 \beta_{3}^{8} \beta_{2}^{2} \beta_{4}^{2}+1449 \beta_{3}^{8} \omega^{4} \beta_{4}^{4}-25920 \beta_{3}^{4} \omega^{2} \beta_{4}^{5} \beta_{2}^{3}\right.\right. \\
& -24192 \beta_{3}^{6} \omega^{2} \beta_{4}^{4} \beta_{2}^{2}+30240 \beta_{3}^{4} \omega^{4} \beta_{4}^{6} \beta_{2}^{2}+324 \beta_{3}^{10} \beta_{2} \beta_{4}-738 \beta_{3}^{10} \omega^{2} \beta_{4}^{2}+1728 \beta_{2}^{3} \beta_{3}^{6} \beta_{4}^{3}-396 \omega^{8} \beta_{3}^{2} \beta_{4}^{9} \beta_{2} \\
& -432 \beta_{2}^{2} \omega^{8} \beta_{4}^{10}+25920 \beta_{3}^{2} \omega^{4} \beta_{4}^{7} \beta_{2}^{3}+504 \beta_{3}^{4} \omega^{6} \beta_{4}^{7} \beta_{2}+11592 \beta_{3}^{6} \omega^{4} \beta_{4}^{5} \beta_{2}-36 \beta_{2} \omega^{10} \beta_{4}^{11}-\beta_{4}^{12} \omega^{12} \\
& \left.-18 \omega^{10} \beta_{3}^{2} \beta_{4}^{10}-7380 \beta_{3}^{8} \omega^{2} \beta_{4}^{3} \beta_{2}\right) \cosh (\omega t)^{3} \\
& +\left(1728 \beta_{3} \omega^{7} \beta_{4}^{9} \beta_{2}^{2}+10368 \beta_{3} \omega^{5} \beta_{4}^{8} \beta_{2}^{3}+12096 \beta_{3}^{3} \omega^{5} \beta_{4}^{7} \beta_{2}^{2}+10368 \beta_{3}^{5} \omega \beta_{4}^{4} \beta_{2}^{3}+5040 \beta_{3}^{5} \omega^{5} \beta_{4}^{6} \beta_{2}\right. \\
& +1440 \beta_{3}^{3} \omega^{7} \beta_{4}^{8} \beta_{2}+288 \beta_{3}^{5} \omega^{7} \beta_{4}^{7}+720 \beta_{3}^{7} \omega^{5} \beta_{4}^{5}-1376 \beta_{3}^{9} \omega^{3} \beta_{4}^{3}+216 \beta_{3}^{11} \omega \beta_{4}+8640 \beta_{3}^{7} \omega \beta_{4}^{3} \beta_{2}^{2} \\
& +72 \beta_{3} \omega^{9} \beta_{4}^{10} \beta_{2}-36288 \beta_{3}^{5} \omega^{3} \beta_{4}^{5} \beta_{2}^{2}-12384 \beta_{3}^{7} \omega^{3} \beta_{4}^{4} \beta_{2}-34560 \beta_{3}^{3} \omega^{3} \beta_{4}^{6} \beta_{2}^{3}+2376 \beta_{3}^{9} \omega \beta_{4}^{2} \beta_{2} \\
& \left.+24 \beta_{3}^{3} \omega^{9} \beta_{4}^{9}\right) \sinh (\omega t) \cosh (\omega t)^{2} \\
& +\mathrm{i}\left(-2304 \beta_{3}^{4} \omega^{6} \beta_{4}^{7} \beta_{2}-2304 \beta_{3}^{8} \omega^{4} \beta_{4}^{4}-3456 \beta_{3}^{2} \omega^{6} \beta_{4}^{8} \beta_{2}^{2}+41472 \beta_{3}^{4} \omega^{2} \beta_{4}^{5} \beta_{2}^{3}-48384 \beta_{3}^{4} \omega^{4} \beta_{4}^{6} \beta_{2}^{2}\right. \\
& +1152 \beta_{3}^{10} \omega^{2} \beta_{4}^{2}-384 \beta_{3}^{6} \omega^{6} \beta_{4}^{6}+38016 \beta_{3}^{6} \omega^{2} \beta_{4}^{4} \beta_{2}^{2}-41472 \beta_{3}^{2} \omega^{4} \beta_{4}^{7} \beta_{2}^{3}+11520 \beta_{3}^{8} \omega^{2} \beta_{4}^{3} \beta_{2} \\
& \left.-18432 \beta_{3}^{6} \omega^{4} \beta_{4}^{5} \beta_{2}\right) \cosh (\omega t)+\left(82944 \beta_{3}^{5} \omega^{3} \beta_{4}^{5} \beta_{2}^{2}+27648 \beta_{3}^{7} \omega^{3} \beta_{4}^{4} \beta_{2}+3072 \beta_{3}^{9} \omega^{3} \beta_{4}^{3}\right. \\
& \left.\left.+82944 \beta_{3}^{3} \omega^{3} \beta_{4}^{6} \beta_{2}^{3}\right) \sinh (\omega t)\right] \exp \left(-\mathrm{i} \frac{\beta_{3}}{\beta_{4}} t\right),
\end{aligned}
$$

The other components of the decomposition series (6) can be determined in a similar way. Substituting (21) into the decomposition series (6), which is a Taylor series, we obtain the closed form solution

$$
\Psi=\Psi_{0}+\Psi_{1}+\Psi_{2}+\Psi_{3}+\Psi_{4}+\ldots=\lambda \operatorname{sech}\left[\frac{\beta_{3} \omega\left(3 \beta_{2} \beta_{4}+\beta_{3}^{2}\right)}{3 \beta_{4}^{2}} z-\omega t\right] \exp \left[\mathrm{i}\left(K z-\frac{\beta_{3}}{\beta_{4}} t\right)\right]
$$

or equivalently

$$
\Psi=\frac{2 \lambda \exp \left[\left(K z-\beta_{3} t / \beta_{4}\right) i+\left(\beta_{3} \omega\left(3 \beta_{2} \beta_{4}+\beta_{3}^{2}\right) z / 3 \beta_{4}^{2}-\omega t\right)\right]}{1+\exp \left[\beta_{3} \omega\left(3 \beta_{2} \beta_{4}+\beta_{3}^{2}\right) z / 3 \beta_{4}^{2}-\omega t\right]^{2}},
$$

with

$$
\omega=\sqrt{\frac{\beta_{4} \lambda^{2}\left(5 \gamma_{2} \lambda^{2}-6 \gamma_{1}\right)}{3 \beta_{3}^{2}+6 \beta_{2} \beta_{4}}}, \quad K=\frac{3 \beta_{3}^{4}-6 \omega^{2} \beta_{3}^{2} \beta_{4}^{2}+12 \beta_{2} \beta_{3}^{2} \beta_{4}-12 \beta_{2} \omega^{2} \beta_{4}^{3}-\beta_{4}^{4} \omega^{4}}{24 \beta_{4}^{3}},
$$

where $\beta_{2}, \beta_{3}, \beta_{4}, \gamma_{1}, \gamma_{2}$ and $\lambda$ are arbitrary constants.

From these values of the pulse parameters, it is simple to see that both the amplitude and the width of the soliton are uniquely determined from the characteristics of the nonlinear medium, i.e. the second- and fourthorder dispersion coefficients and the two nonlinear coefficients. 
For comparison, we consider another initial condition of (1) as

$$
\Psi(t, 0)=\sqrt{-\frac{2 \gamma_{1}}{5 \gamma_{2}}}[\operatorname{JacobiSN}(-\varpi t, m)+\operatorname{JacobiCN}(-\varpi t, m)] \exp \left(-\mathrm{i} \frac{\beta_{3}}{\beta_{4}} t\right)
$$

with $\varpi=\sqrt{\left(12 \beta_{2} \beta_{4}+6 \beta_{3}^{2}\right) /\left(10 \beta_{4}^{2}-5 \beta_{4}^{2} m^{2}\right)}$, where JacobiSN $(-\varpi t, m)$ and JacobiCN $(-\varpi t, m)$ are the Jacobian elliptic sine function and cosine function. They are periodic with period $2 K(m)$, where $K(m), K(m)=$ $\int_{0}^{\pi / 2} \mathrm{~d} x / \sqrt{1-m^{2} \sin ^{2} x}$, is the complete elliptic integral of the first kind, $\beta_{2}, \beta_{3}$ and $\beta_{4}$ are arbitrary constants, and the coefficients $\gamma_{1}, \gamma_{2}$ must have opposite signs $\left(\gamma_{1} \gamma_{2}<0\right)$.

Rewriting (1) for initial condition (25) in a operator form as (3), then using (9) and (10) with (11), one can construct the terms of the decomposition series. Some of the terms of the series are as follows:

$$
\begin{aligned}
& \Psi_{0}=\sqrt{-\frac{2 \gamma_{1}}{5 \gamma_{2}}}[\operatorname{JacobiSN}(-\varpi t, m)+\operatorname{JacobiCN}(-\varpi t, m)] \exp \left(-\mathrm{i} \frac{\beta_{3}}{\beta_{4}} t\right), \\
& \Psi_{1}(t, z)=\int_{0}^{z}\left[-\mathrm{i} \frac{\beta_{2}}{2} L_{2, t} \Psi_{0}+\frac{\beta_{3}}{6} L_{3, t} \Psi_{0}-\mathrm{i} \frac{\beta_{4}}{24} L_{4, t} \Psi_{0}+\gamma_{1} A_{1,0}+\gamma_{2} A_{2,0}\right], \ldots, \\
& \Psi_{n+1}(t, z)=\int_{0}^{z}\left[-\mathrm{i} \frac{\beta_{2}}{2} L_{2, t} \Psi_{n}+\frac{\beta_{3}}{6} L_{3, t} \Psi_{n}-\mathrm{i} \frac{\beta_{4}}{24} L_{4, t} \Psi_{n}+\gamma_{1} A_{1, n}+\gamma_{2} A_{2, n}\right],
\end{aligned}
$$

where $n \geq 1$, and the Adomian polynomials $A_{i, n},(i=1,2)$ are the same as in the formulae (11). Performing the calculations in (26) with (11) using Maple and substituting them into (6) give the exact solution

$$
\Psi(t, z)=\sqrt{-\frac{2 \gamma_{1}}{5 \gamma_{2}}}[\operatorname{JacobiSN}(k z-\varpi t, m)+\operatorname{JacobiCN}(k z-\varpi t, m)] \exp [\mathrm{i}(K z-\Omega t)]
$$

with

$$
\Omega=\frac{\beta_{3}}{\beta_{4}}, \quad k=\frac{\beta_{3} \varpi\left(3 \beta_{2} \beta_{4}+\beta_{3}^{2}\right)}{3 \beta_{4}^{2}}
$$

and

$$
\varpi=\sqrt{\frac{12 \beta_{2} \beta_{4}+6 \beta_{3}^{2}}{10 \beta_{4}^{2}-5 \beta_{4}^{2} m^{2}}}, \quad K=\frac{-3 \beta_{3}^{4}+\varpi^{2}\left(9 \beta_{4}^{4} \varpi^{2} m^{4}+10 \beta_{3}^{2} \beta_{4}^{2}-5 \beta_{3}^{2} \beta_{4}^{2} m^{2}+9 \beta_{4}^{4} \varpi^{2}-9 \beta_{4}^{4} \varpi^{2} m^{2}\right)}{24 \beta_{4}^{3}},
$$

where $\beta_{2}, \beta_{3}, \beta_{4}, \gamma_{1}$ and $\gamma_{2}$ are arbitrary constants.

When $m=1$, the solution (27) degenerates to

$$
\Psi(t, z)=\sqrt{-\frac{2 \gamma_{1}}{5 \gamma_{2}}}[\operatorname{sech}(k z-\varpi t)+\tanh (k z-\varpi t)] \exp (\mathrm{i} K z-\mathrm{i} \Omega t)
$$

with (28) and

$$
K=-\frac{3 \beta_{3}^{4}-9 \beta_{4}^{4} \varpi^{4}-5 \varpi^{2} \beta_{3}^{2} \beta_{4}^{2}}{24 \beta_{4}^{3}}, \quad \varpi=\sqrt{\frac{12 \beta_{2} \beta_{4}+6 \beta_{3}^{2}}{5 \beta_{4}^{2}}} .
$$

In fact, we also obtain the result (30) from the initial condition

$$
\Psi(t, 0)=-\sqrt{-\frac{2 \gamma_{1}}{5 \gamma_{2}}} \frac{[\exp (2 \varpi t)-1-2 \exp (\varpi t)]}{[\exp (2 \varpi x)+1]} \exp \left(-\mathrm{i} \frac{\beta_{3}}{\beta_{4}} t\right), \varpi=\sqrt{\frac{12 \beta_{2} \beta_{4}+6 \beta_{3}^{2}}{5 \beta_{4}^{2}}} .
$$

It is obvious from the above expression that the coefficients must be satisfy the restriction $\beta_{3}^{2} \geq-2 \beta_{2} \beta_{4}$. 
(a)

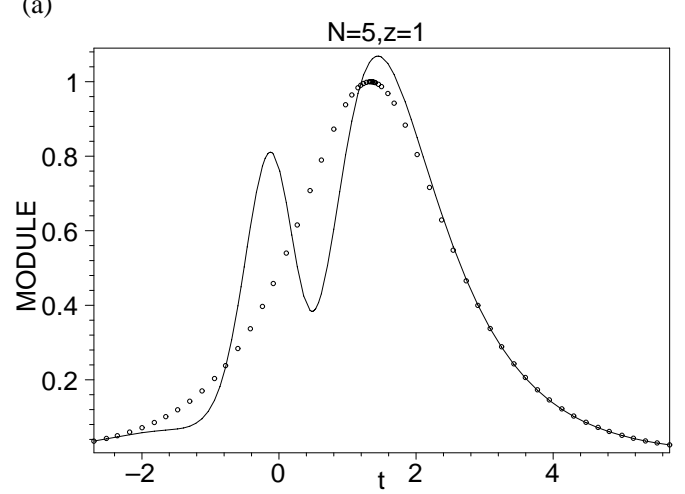

(c)

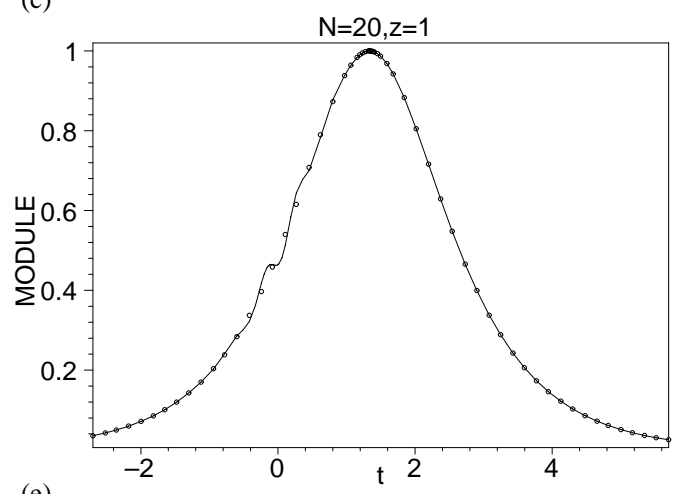

(e)

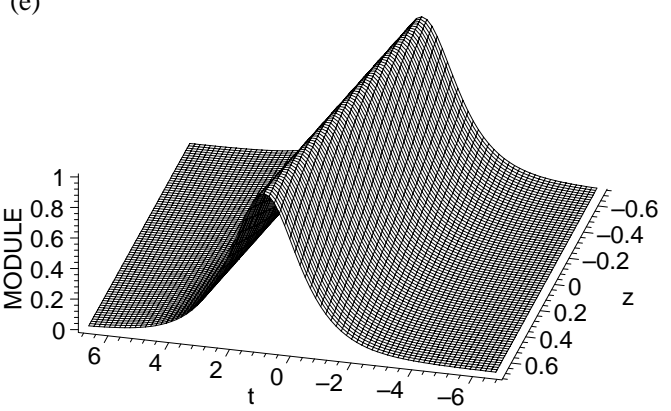

\section{Numerical Results of the ADM}

For numerical comparisons purposes, we construct the solution $\Psi(t, z)$ as

$$
\Psi(t, z)=\lim _{N \rightarrow \infty} \varphi_{N},
$$

where $\varphi_{N}(t, z)$, the $N$-term approximation for $\Psi(t, z)$, is a finite series defined as

$$
\varphi_{N}(t, z)=\sum_{n=0}^{N-1} \Psi_{n}(t, z), \quad N \geq 1
$$

and the recurrence relation is given as in (10) with (11). It is worth pointing out that the advantage of the decomposition methodology is the fast convergence of (b)

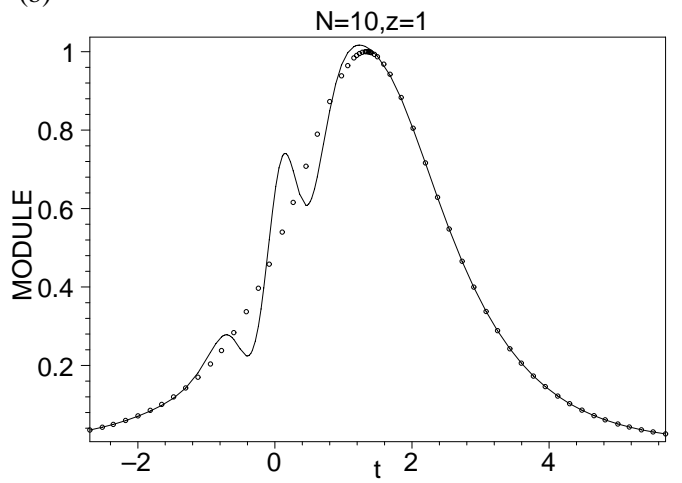

(d)

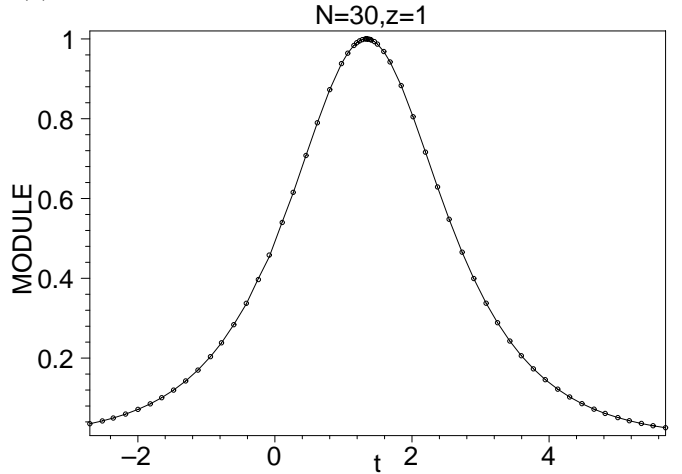

Fig. 1. (a)-(d) The module graphs of the exact (point) result (22) $|\Psi|$ and approximate (line) result $\left|\varphi_{n}\right|$ with the initial condition (17) at (a) $N=5, z=1$; (b) $N=10, z=1$; (c) $N=20, z=1$; (d) $N=30, z=1$. (e) The surface shows the module of numerical result $\varphi_{30}$ for $-6.7 \leq t \leq 6.7$ and $-0.7 \leq z \leq 0.7$.

Table 1. Numerical results (in $z$ direction) for modules of exact result $|\Psi(t, z)|$, approximate result $\left|\varphi_{30}(t, z)\right|$, and absolute error $\left|\Psi(t, z)-\varphi_{30}(t, z)\right|$, where $\Psi(t, z)=\operatorname{sech}\left(\frac{4}{3} z-\right.$ $t) \exp \left[\mathrm{i}\left(-\frac{z}{6}-t\right)\right]$ for $(1)$.

\begin{tabular}{lccl}
\hline$\left(t_{i}, z_{i}\right)$ & $\begin{array}{c}\text { Exact } \\
\text { solution }|\Psi|\end{array}$ & $\begin{array}{c}\text { Approximate } \\
\text { solution }\left|\varphi_{30}\right|\end{array}$ & $\begin{array}{c}\text { Absolute } \\
\text { error }\left|\Psi-\varphi_{30}\right|\end{array}$ \\
\hline$(0.01,0.01)$ & 0.9999944440 & 0.9999944447 & $7.000000 \mathrm{E}-10$ \\
$(0.02,0.02)$ & 0.9999777785 & 0.9999777775 & $1.000000 \mathrm{E}-9$ \\
$(0.02,0.03)$ & 0.9998000330 & 0.9998000336 & $6.000000 \mathrm{E}-10$ \\
$(0.03,0.04)$ & 0.9997278391 & 0.9997278396 & $5.000000 \mathrm{E}-10$ \\
$(0.05,0.05)$ & 0.9998611273 & 0.9998611270 & $3.000000 \mathrm{E}-10$ \\
$(0.04,0.01)$ & 0.9996445494 & 0.9996445491 & $3.000000 \mathrm{E}-10$ \\
$(0.05,0.02)$ & 0.9997278391 & 0.9997278388 & $3.000000 \mathrm{E}-10$ \\
$(0.04,0.03)$ & 1.0000000000 & 0.9999999995 & $5.000000 \mathrm{E}-10$ \\
$(0.05,0.04)$ & 0.9999944440 & 0.9999944450 & $1.000000 \mathrm{E}-9$ \\
$(0.05,0.05)$ & 0.9998611273 & 0.9998611274 & $1.000000 \mathrm{E}-10$ \\
\hline
\end{tabular}


(a)

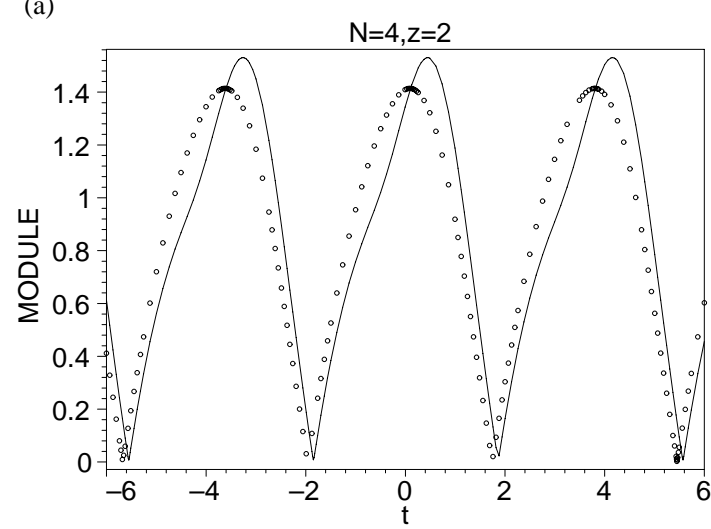

(c)

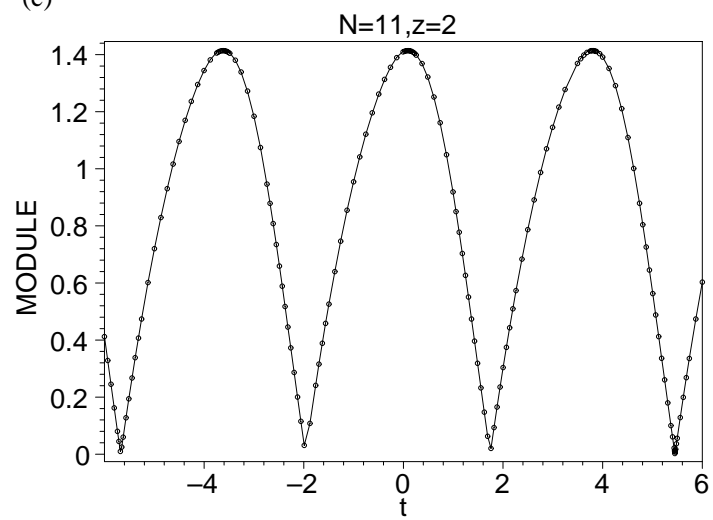

(b)

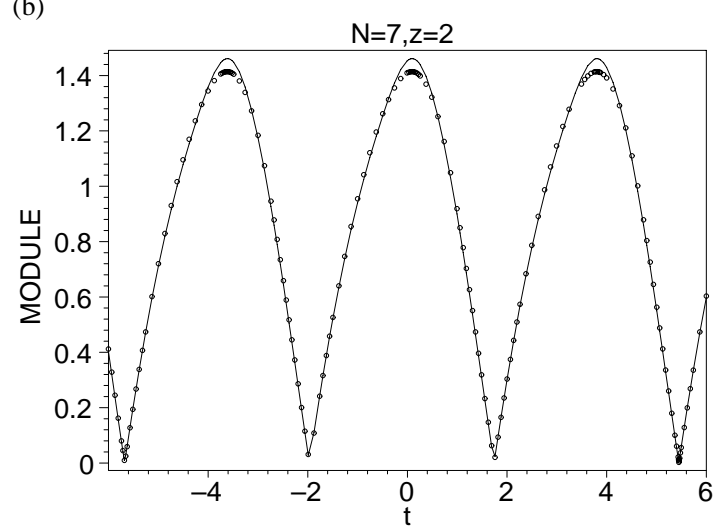

(d)

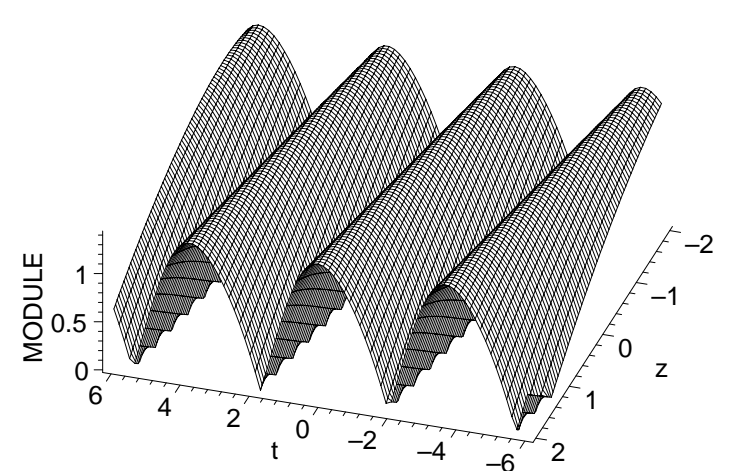

Fig. 2. (a) - (c) The module graphs of the exact (point) result (27) $|\Psi|$ and approximate (line) result $\left|\varphi_{n}\right|$ with the initial condition (25) at (a) $N=4, z=2$; (b) $N=7, z=2$; (c) $N=11, z=2$, respectively, where $m=\frac{\sqrt{2}}{2}$. (d) The surface shows the module of numerical result $\left|\varphi_{11}\right|$ with the initial condition (25) and $m=\frac{\sqrt{2}}{2}$ for $-6 \leq t \leq 6$ and $-2 \leq z \leq 2$.

(a)

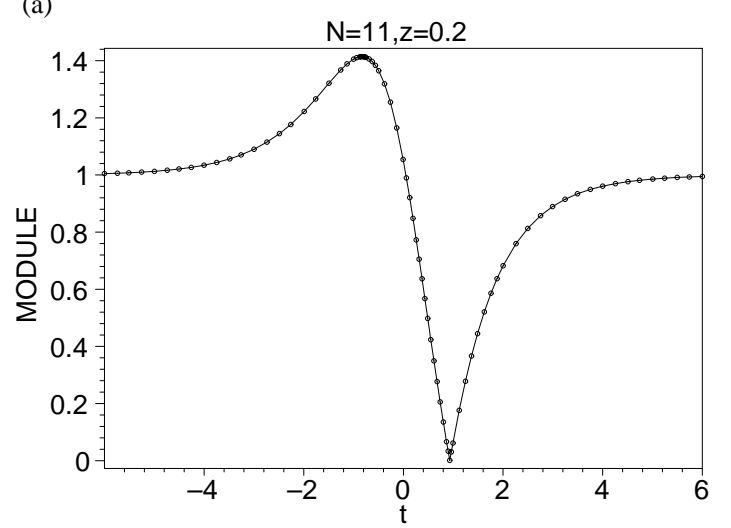

(b)

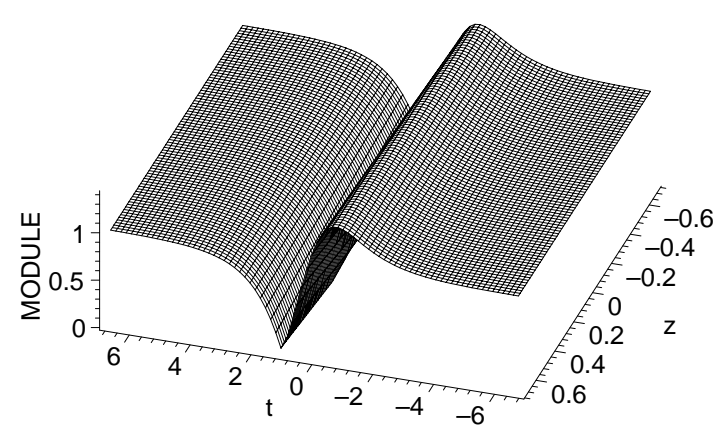

Fig. 3. (a) The module graph of the exact (point) result (30) $|\Psi|$ and approximate (line) result $\left|\varphi_{11}\right|$ with the initial condition (32), where $z=0.2$. (b) The surface shows the module of numerical solution $\left|\varphi_{11}\right|$ of (1) for $-6.7 \leq t \leq 6.7$ and $-0.7 \leq z \leq 0.7$.

the solutions in real physical problems [24]. The theoretical treatment of convergence of the decomposition method has been considered in the literature [24-26]. The obtained results about the speed of convergence of 
Table 2. Numerical results (in $z$ direction) for modules of absolute error $\left|\Psi(t, z)-\varphi_{30}(t, z)\right|$, where $\Psi(t, z)=$ $\left[\operatorname{JacobiSN}\left(\frac{11}{24} z-t, \frac{\sqrt{2}}{2}\right)+\operatorname{JacobiCN}\left(\frac{11}{24} z-t, \frac{\sqrt{2}}{2}\right)\right] \exp \left(\mathrm{i} \frac{15}{32} z-\mathrm{i} t\right)$ for (1).

\begin{tabular}{cccccc}
\hline$t_{i}$ & 0.01 & 0.02 & $z_{i}$ & 0.04 & 0.05 \\
\hline 0.01 & $1.0000 \mathrm{E}-10$ & $2.0000 \mathrm{E}-10$ & $1.0000 \mathrm{E}-9$ & $1.0000 \mathrm{E}-9$ & $0.0000 \mathrm{E}+00$ \\
0.02 & $1.0000 \mathrm{E}-10$ & $9.0000 \mathrm{E}-10$ & $1.1000 \mathrm{E}-9$ & $7.1000 \mathrm{E}-10$ & $0.0000 \mathrm{E}+00$ \\
0.03 & $5.0000 \mathrm{E}-10$ & $7.0000 \mathrm{E}-10$ & $6.0000 \mathrm{E}-10$ & $4.0000 \mathrm{E}-10$ & $1.2000 \mathrm{E}-9$ \\
0.04 & $4.0000 \mathrm{E}-10$ & $4.0000 \mathrm{E}-10$ & $7.0000 \mathrm{E}-10$ & $1.0000 \mathrm{E}-10$ & $1.0000 \mathrm{E}-10$ \\
0.05 & $5.0000 \mathrm{E}-10$ & $3.0000 \mathrm{E}-10$ & $0.0000 \mathrm{E}+00$ & $3.0000 \mathrm{E}-10$ & $0.0000 \mathrm{E}+00$ \\
\hline
\end{tabular}

this method were enabling us to solve linear and nonlinear functional equations. In a recent work Ngarhasta et al. [33] have proposed a new approach of convergence of the decomposition series. The authors have given a new condition for obtaining convergence of the decomposition series to the classical presentation of the ADM.

Moreover, as the decomposition method does not require discretization of the variables time and space, it is not effected by computation round off errors and one is not faced with the necessity of large computer memory and time. The accuracy of the decomposition method for (1) is controllable and absolute errors $\left|\Psi-\varphi_{N}\right|$ are very small with the present choice of $t$ and $z$ which are given in Tables 1 and 2 . In most cases $\varphi_{N}$ is accurate for quite low values of $N$. For initial condition (25), we achieve a very good approximation to the partial exact solution by using only 11 terms of the decomposition series, which shows that the speed of convergence of this method is very fast. It is evident that the overall errors can be made smaller by adding new terms of the decomposition series. Both the exact results and the approximate solutions obtained by using the formulae (33) with (34) are plotted in Figs. 1, 2 and 3 for (1) with different initial conditions (17), (25) and (32), respectively. It is evident that when computing more terms for the decomposition series the numerical results are getting much more closer to the corresponding exact solutions with the initial conditions of (1).

\section{Conclusion}

In this paper, we considered a numerical treatment for the solution of the HDCNS equation using the ADM. To the best of our knowledge, this is the first result on the application of the ADM to this equation. This method transforms (1) into a recursive relation.

The obtained numerical results compared with the analytical solution show that the method provides re- markable accuracy especially for small values of the space $z$. Generally speaking, the ADM provides analytic, verifiable, rapidly convergent approximation which yields insight into the character and the behavior of the solution just as in the closed form solution. It solves nonlinear problems without requiring linearization, perturbation, or unjustified assumptions which may change the problem being solved. The method can also easyly be extended to other similar physical equations, with the aid of Maple (or Matlab, Mathematica, etc.), the course of solving nonlinear evaluation equations can be carried out in a computer.

As we known, although the decomposition series (6) obtained by using ADM is infinite, we often replace the exact solution with a finite series

$$
\varphi_{N}(t, z)=\sum_{n=0}^{N-1} \Psi_{n}(t, z)
$$

which is quickly convergent towards the accurate solution for quite low values of $N$. On this account, there is a common phenomenon in the related literature [24-32]. It can easily be noted, that no matter whether the examples are from the related literature or from this paper, the space or time variables in the pictures are all taken in small scales. Since the Taylor series method provides the same answer obtained by the ADM, we can proceed from the nature of the Taylor series $[34,35]$ to study this phenomenon. The Taylor series expansion of the function $\Psi(t, z)$ about $z=z_{0}$ is given by

$$
\Psi(t, z)=\sum_{n=0}^{\infty} \frac{\Psi^{(n)}\left(t, z_{0}\right)}{n !}\left(z-z_{0}\right)^{n}
$$

or, equivalently

$\Psi(t, z)=\sum_{n=0}^{N-1} \frac{\Psi^{(n)}\left(t, z_{0}\right)}{n !}\left(z-z_{0}\right)^{n}+R_{N-1}, N \geq 1$.

Here, $R_{N-1}$ is a remainder term known as the Lagrange 
(a)

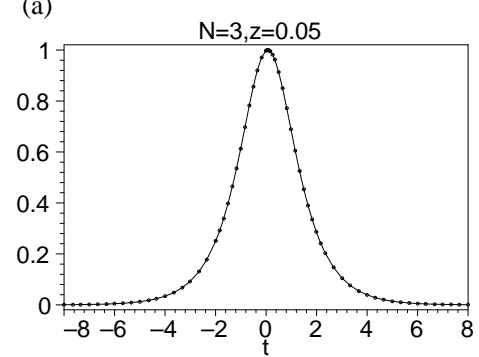

(b)

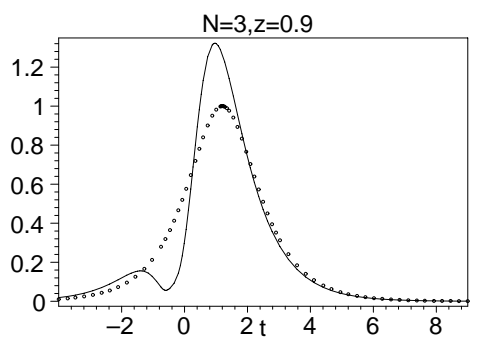

(c)

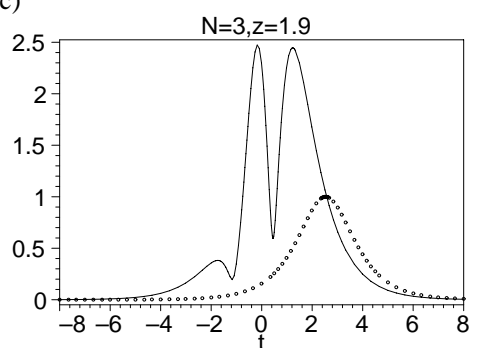

Fig. 4. The module graph of the exact (point) result (22) $|\Psi|$ and approximate (line) result $\left|\varphi_{3}\right|$ with the initial condition (17) of (1) at different values of $z$.

(a)

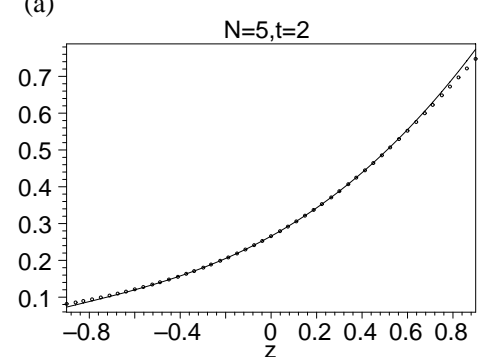

(b)

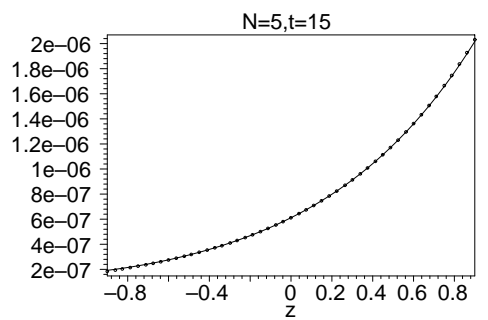

(c)

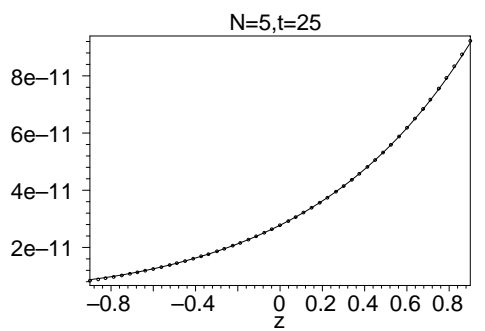

Fig. 5. The module graphs of the exact (point) result (22) $|\Psi|$ and approximate (line) result $\left|\varphi_{5}\right|$ with the initial condition (17) at different values of $t$.

remainder, which is given by

$$
\begin{aligned}
R_{N-1} & =\underbrace{\int \cdots \int_{z_{0}}^{z}}_{N} \Psi^{(N)}(t, z)(\mathrm{d} z)^{N} \\
& =\frac{\left(z-z_{0}\right)^{N}}{(N) !} \Psi^{(N)}\left(z^{*}\right), \quad z^{*} \in\left[z_{0}, z\right] .
\end{aligned}
$$

As we known, the decomposition series (6) is exactly a Taylor series of exact solution $\Psi$ about a point $z=0$, that is

$$
\varphi_{N}=\sum_{n=0}^{N-1} \Psi_{n}(t, z)=\sum_{n=0}^{N-1} \frac{\Psi^{(n)}(t, 0)}{n !} z^{n}, N \geq 1 .
$$

Then the remainder term $R_{N-1}$, i.e. the error between analytical and approximate solutions, is

$$
R_{N-1}=\Psi-\sum_{n=0}^{N-1} \frac{\Psi^{(n)}(t, 0)}{n !} z^{n}=\Psi-\varphi_{N}
$$

[1] D. Anderson and M. Lisak, Phys. Rev. A 27, 1393 (1983).

[2] G. P. Agrawal and C. Headley, Phys. Rev. A 46, 1573 (1992).

$$
\begin{aligned}
& =\underbrace{\int \cdots \int_{0}^{z}}_{N} \Psi^{(N)}(t, z)(\mathrm{d} z)^{N}=\frac{(z)^{N}}{N !} \Psi^{(N)}\left(z^{*}\right), \\
& z^{*} \in[0, z], N \geq 1
\end{aligned}
$$

which can be obtained by using the mean-value theorem. From the formula above, we know that the greater the fetching value of $|z|$ ( $z$ is farther and farther from the point $z=0$ ) is, the greater is the error $R_{N-1}$ (see Fig. 4), although the approximated solution can be calculated for any $t$ and $z$. Nearing zero for $z$, the approximate solution is almost according to the exact solution at any value of time $t$ (see Fig. 5). From Figs. $1-5$ one might find out that both the term number $N$ and the value of $z$ influence the approximation precision of the numerical solution (34) for the corresponding exact solution of the HDCNS equation, whereas time $t$ has only a little effect on this.

[3] D. Mihalache, N. Truta, and L. C. Crasovan, Phys. Rev. E 56, 3955 (1996).

[4] M. Gedalin, T.C. Scott, and Y.B. Band, Phys. Rev. Lett. 78, 448 (1997). 
[5] A.D. Tatiana and A.Z. Yuri, Physica D 156, 260 (2001).

[6] L.P. Sergio, Chaos, Solitons and Fractals 19, 203 (2004).

[7] L. D. Hai, Wave Motion 33, 339 (2001).

[8] P. Honzatko, Opt. Commun. 127, 363 (1996).

[9] G. M. Muslu and H. A. Erbay, Math. Comput. Simulation 57, 581 (2005).

[10] H. R. Yu, L. Lu, L. Z. Hao, Y. R. Cao, and Z. G. Sheng, Opt. Commun. 245, 383 (2005).

[11] S.L. Palacios and J.M. Fernandez-Diaz, Opt. Commun. 178, 457 (2000).

[12] W. P. Hong, Opt. Commun. 213, 173 (2002).

[13] S. L. Palacios and J. M. Fernandez-Diaz, J. Mod. Opt. 48, 1691 (2001).

[14] A. G. Shagalov, Phys. Lett. A 239, 41 (1998).

[15] V. I. Karpman, Phys. Lett. A 244, 397 (1998).

[16] V. I. Karpman and A. G. Shagalov, Phys. Lett. A 254, 319 (1999).

[17] S. M. El-Sayed and D. Kaya, Appl. Math. Comput. (in press).

[18] M. A. Abdou and A. A. Soliman, Physica D: Nonlinear Phenomena (in press).

[19] D. Kaya and S. M. El-Sayed, Phys. Lett. A 313, 82 (2003).

[20] S. A. Khuri, Appl. Math. Comput. 97, 251 (1998).

[21] G. Adomian, Appl. Math. Comput. 88, 127 (1997).
[22] G. Adomian and R. E. Meyers, Appl. Math. Lett. 8, 7 (1995).

[23] B. K. Datta, Comput. Math. Appl. 20, 61 (1990).

[24] G. Adomian, R. Rach, and N.T. Shawagfeh, Found. Phys. Lett. 8, 161 (1995).

[25] G. Adomian and R. Rach, Appl. Math. Comput. 24, 61 (1992).

[26] A. M. Wazwaz, Partial Differential Equations: Methods and Applications, Balkema, Rotterdam 2002.

[27] S. V. Tonningen, Comput. Educ. J. 5, 30 (1995).

[28] R. Rach, J. Math. Anal. Appl. 128, 480 (1987).

[29] J. Y. Edwards, J. A. Roberts, and N. J. Ford, Technical Report No. 309, Manchester Center of Computational Mathematics, Manchester 1997, p. 1 C17.

[30] A. M. Wazwaz, Appl. Math. Comput. 79, 37 (1998).

[31] M. E. Salah and R. A. Mohammedi, Appl. Math. Comput. 136, 151 (2003).

[32] T. Geyikli and D. Kaya, Appl. Math. Comput. 169, 146 (2005).

[33] N. Ngarhasta, B. Some, K. Abbaoui, and Y. Cherruault, Kybernetes 31, 61 (2002).

[34] M. Abramowitz and I. A. Stegun, Handbook of Mathematical Functions with Formulas, Graphs, and Mathematical Tables, 9th ed., Dover, New York 1972, p. 880.

[35] G. Arfken, Taylor's Expansion, in: Mathematical Methods for Physicists, 3rd. ed., Academic Press, Orlando, FL 1985, pp. $303-313$. 